\title{
Caso Clínico 02-2019. Varón de 55 años con alteración de la conciencia, movimientos involuntarios y baja de peso
}

Clinical case 02-2019. 55 years old man with altered consciousness, involuntary movements and weight loss

\author{
Héctor Sosa ${ }^{1, a}$, Milton Blanco ${ }^{1, b}$
}

\section{PRESENTACIÓN DEL CASO}

\section{Milton Blanco (Residente de 3er año de Medicina Interna):}

Varón de 55 años, natural de Moquegua y procedente de Chosica, Departamento de Lima, con una historia de 5 meses de inicio insidioso, curso crónico, caracterizado por disgeusia, movimientos involuntarios y confusión.

Cinco meses antes del ingreso refiere alteración de los sabores de forma intermitente que luego se hace persistente. Dos meses antes del ingreso, nota baja de peso, de aproximadamente $8 \mathrm{~kg}$ de forma involuntaria. Quince días después refiere presencia de movimientos involuntarios repetitivos de miembros inferiores que le impiden la deambulación, en tres oportunidades, siempre después de levantarse en las mañanas y que ceden post ingesta del desayuno. Un mes después mediante consulta ambulatoria le diagnostican probable deficiencia de zinc y le indican suplementación.

Dos semanas antes del ingreso, la disgeusia no mejora y busca nuevamente atención médica; en esta ocasión le informan que debe realizarse una biopsia de labio por sospecha de amiloidosis o síndrome de Sjögren, la cual se realiza, siendo el resultado negativo.
Cuatro días antes del ingreso, en la mañana, momentos antes de realizarse una colonoscopia programada, el familiar lo nota confuso, hablando incoherencias con agitación psicomotrizy movimientos involuntarios; le diagnostican síndrome conversivo y le dan de alta. Sin embargo, al persistir estos síntomas es llevado a emergencia de otro nosocomio donde se le encuentra glicemia capilar en $28 \mathrm{mg} / \mathrm{dl}$, le administran soluciones glucosadas con mejoría notable y se le da el alta. Estando asintomático decide acudir por consultorio de medicina interna de nuestra institución, decidiéndose su hospitalización.

El apetito se encontraba disminuido, la sed aumentada, el sueño y la sudoración conservada, había una marcada disminución de peso (17 kilos en los últimos cinco meses), deposiciones sin alteraciones y estado de ánimo distímico.

El paciente vivía en Lima desde hace varios años, negaba viajes durante este último año, era docente y negaba crianza de animales.

Como antecedentes refería diagnóstico de tuberculosis pulmonar hace 40 años por el cual recibió tratamiento completo, fractura de clavícula izquierda secundario a accidente de moto, negaba eliminación de parásitos, refería vacunación contra fiebre amarilla hace 3 años, había tenido dos parejas sexuales y además negaba hábitos nocivos.

\footnotetext{
Servicio de Medicina Interna, Departamento de Medicina, Hospital Nacional Cayetano Heredia. Ministerio de Salud. Lima, Perú. Médico asistente

Médico residente
} 
Su padre había fallecido de infarto agudo de miocardio, su madre era diabética, hipertensa y tenía glaucoma, tenía una hermana con neoplasia intrabdominal y otra con diabetes mellitus.

En la revisión anamnésica de aparatos y sistemas refería eventualmente dolor en las rodillas que empeoraban con el movimiento y mejoraban con el reposo.

En el examen físico al ingreso tenía una presión arterial de 120/70, frecuencia cardiaca 76 latidos/min, frecuencia respiratoria 15 respiraciones por minuto, afebril, saturación $98 \%$ a FiO 2 ambiental, peso $65 \mathrm{~kg}$ e IMC de $24 \mathrm{~kg} / \mathrm{m}^{2}$.

Estaba en regular estado general, regular estado de hidratación, regular estado nutricional, en decúbito dorsal activo, piel tibia, hidratada, llenado capilar < 2 segundos, no había palidez ni edema, tenía facies seborreica con acentuación de pliegues frontales y acropaquias con uñas en vidrio de reloj (figura 1), no había alteración en las articulaciones, ni se palpaban adenopatías. En el tórax se encontró abolición de las vibraciones vocales y del murmullo vesicular en los
2/3 inferiores de hemitórax derecho, el otro campo pulmonar sin alteraciones; en aparato cardiovascular los ruidos cardiacos eran rítmicos, no se encontraron soplos, ingurgitación yugular ni reflujo hepatoyugular, los pulsos estaba preservados; el abdomen era blando no había dolor a la palpación superficial ni profunda, no se encontró visceromegalia; al examen neurológico estaba despierto, con escala de Glasgow 15/15, fuerza y reflejos sin anormalidades, sin focalización ni signos meníngeos, resto sin anormalidades, fondo de ojo no contributorio.

Los exámenes de laboratorio al ingreso mostraron: hemoglobina 13,4 g/dl, hemograma y recuento plaquetario sin alteraciones, urea, creatinina, electrolitos, calcio iónico, perfil hepático, TP y TTP dentro de lo normal; velocidad de sedimentación en 53 $\mathrm{mm} / \mathrm{h}(\mathrm{VN} 0$ a 20$)$, TSH en $1,49 \mathrm{uU} / \mathrm{ml}(\mathrm{VN} 0,4$ a 4$)$ y $\mathrm{T}_{4}$ libre en $1,19 \mathrm{ng} / \mathrm{dl}(\mathrm{VN} 0,7$ a 1,8).

Una hora después del ingreso el paciente se tornó agitado, confuso y agresivo, se le tomó una glucosa capilar resultando en $31 \mathrm{mg} / \mathrm{dl}$; se administró $30 \mathrm{~g}$ de dextrosa en bolo endovenoso y continuó con infusión de dextrosa al $10 \%$, luego de cual el paciente se

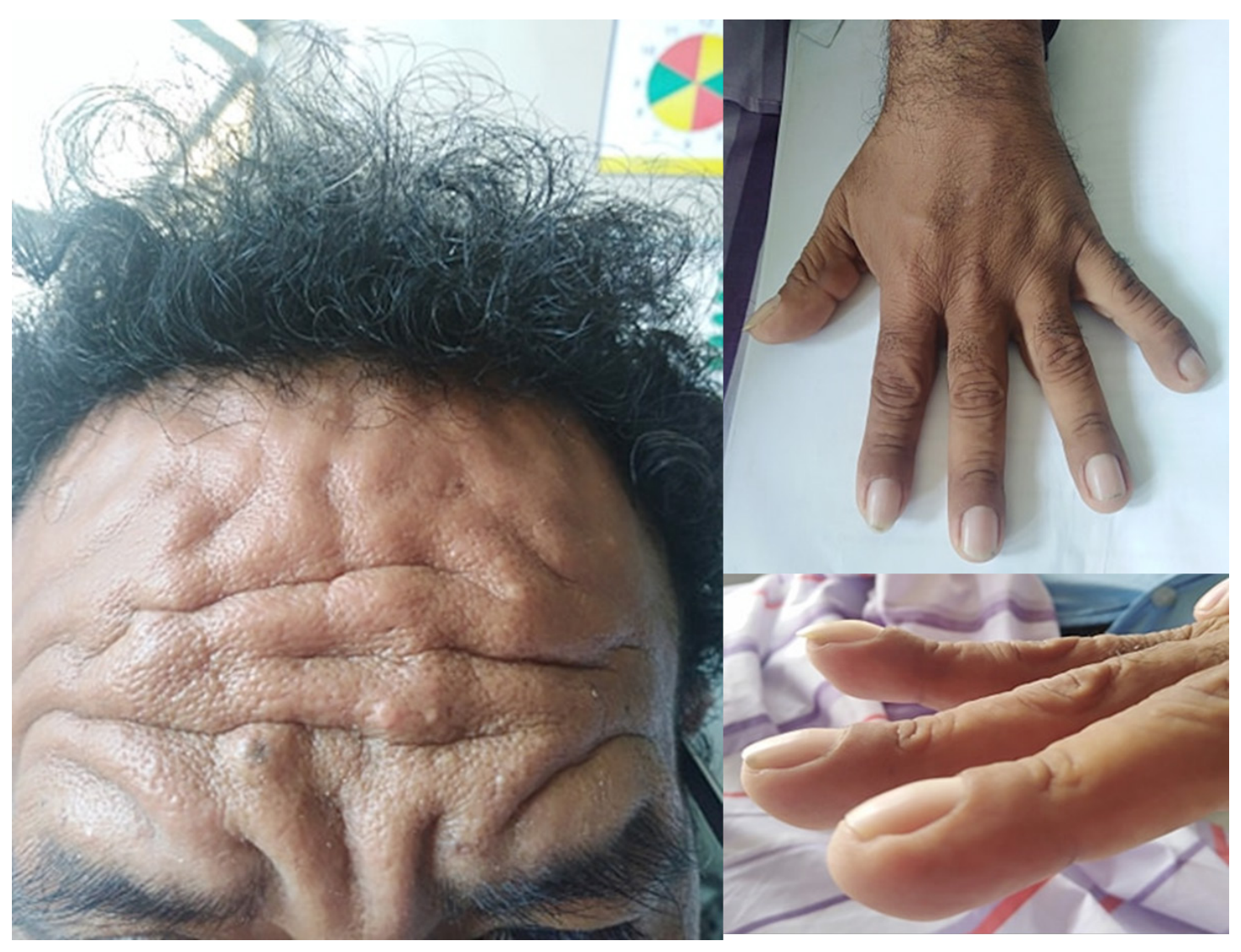

Figura 1. Acentuación de pliegues frontales y acropaquias con uñas en vidrio de reloj. 


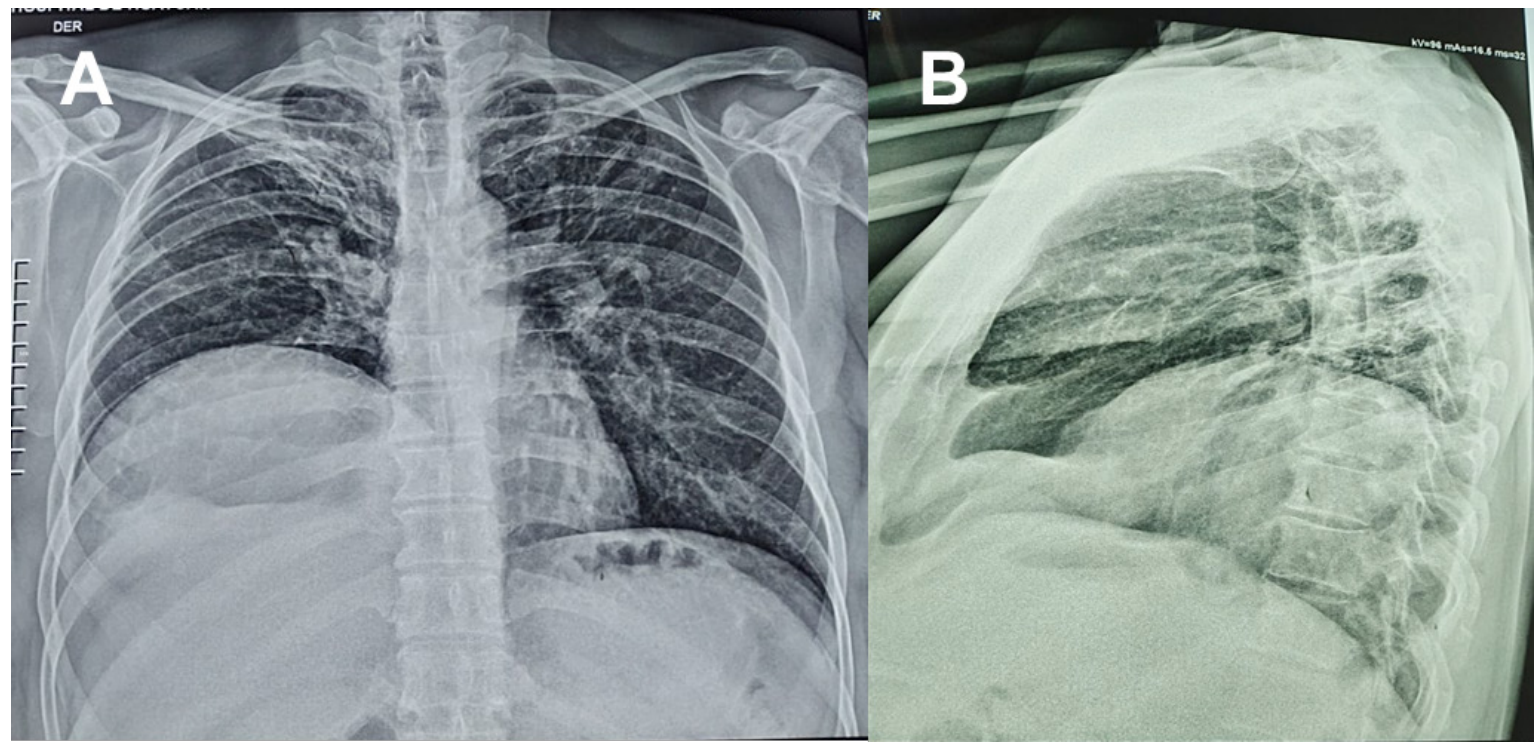

Figura 2. Radiografía de tórax, muestra opacidad en base pulmonar derecha posterior.

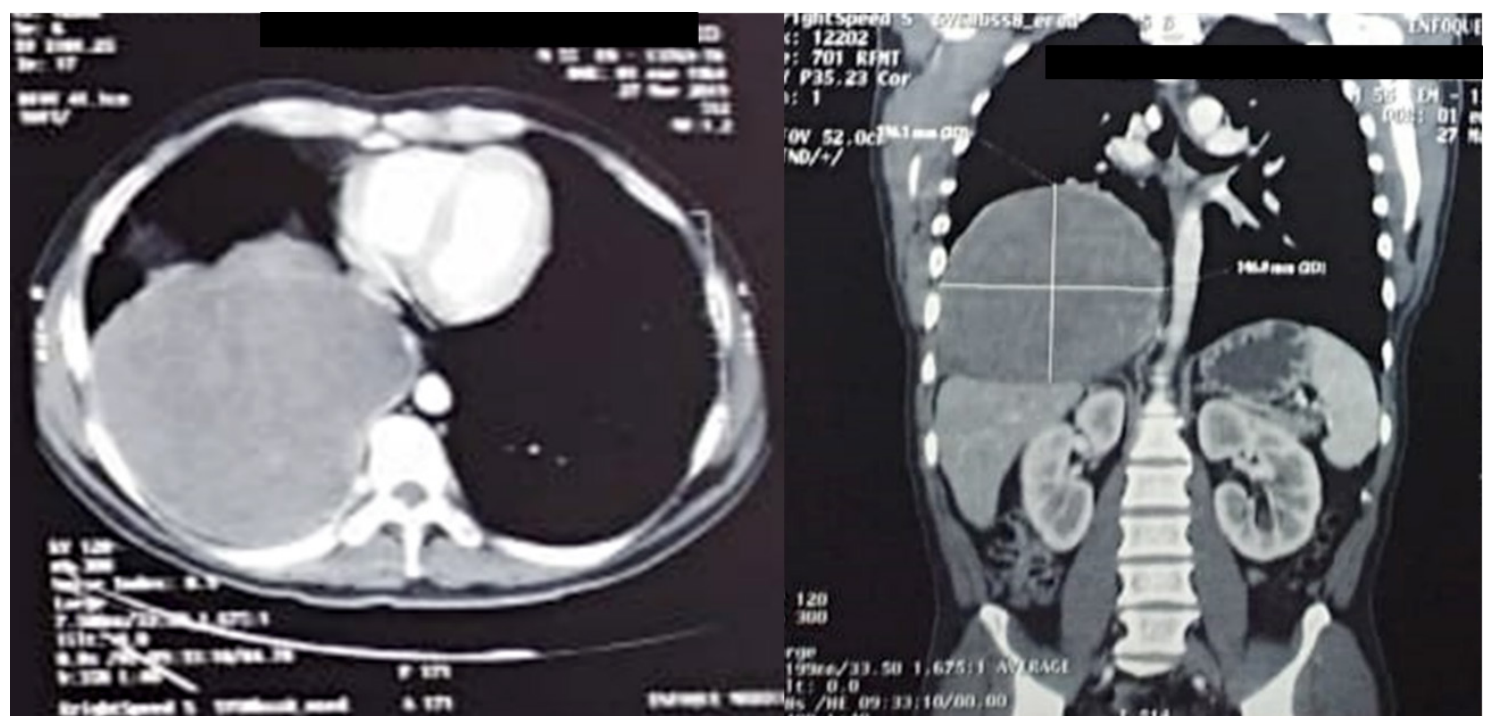

Figura 3. Tomografía que muestra tumoración pulmonar derecha redondeada que ocupa casi la totalidad del lóbulo inferior derecho, de bordes definidos con escasas calcificaciones lineales discontinuas y con aparentes septos y loculaciones internas, tractos fibro retráctiles con calcificaciones y dilataciones bronquiales de tracción en segmentos apicales.

recuperó mejorando completamente el estatus mental; la glucosa capilar control fue $172 \mathrm{mg} / \mathrm{dl}$.

El electrocardiograma mostró ritmo sinusal, eje normal, sin anormalidades. La radiografía de tórax postero-anterior reveló una radiopacidad en base pulmonar derecha posterior (figura $2 \mathrm{a}$ y $2 \mathrm{~b}$ ).

En la tomografía de tórax y abdomino pélvica con contraste, se evidenció una masa pulmonar derecha redondeada de $149 \mathrm{~mm}$ x $142 \mathrm{~mm}$, que ocupaba casi la totalidad del lóbulo inferior derecho, de bordes definidos con escasas calcificaciones lineales discontinuas y densidad de 33 a $35 \mathrm{UH}$, con aparentes septos y loculaciones internas, tractos fibro retráctiles con calcificaciones y dilataciones bronquiales de tracción en segmentos apicales. No había adenomegalias y el resto se encontraba dentro la normalidad (figura 3). La tomografía abdomino pélvica solo evidenció litiasis renal bilateral y diverticulosis colónica, el resto sin anormalidades. 


\section{DISCUSIÓN CLÍNICA}

\section{Dr. Héctor Sosa (Medicina Interna)}

Nos encontramos ante un paciente con una historia crónica de movimientos involuntarios ocasionales pero recurrentes, que por lo demás entre estos episodios, esta asintomático. Estos episodios tienen la particularidad de manifestarse sin alteración de la conciencia, son matutinos y ceden con la ingesta del desayuno. A todo esto, se le añade un nuevo episodio de movimientos involuntarios que se acompaña de trastorno del sensorio, todo esto antes de un procedimiento colonoscópico (para lo cual tuvo un ayuno prolongado), en esa ocasión se le encontró glucosa en $28 \mathrm{mg} / \mathrm{dl}$ por lo que se le administró glucosa endovenosa, con remisión completa de los síntomas.

Lo primero que habría que comentar es que este paciente tiene un diagnóstico establecido de hipoglicemia pues cumple los criterios de la triada de Whipple: glucosa en sangre menos de $45 \mathrm{mg} / \mathrm{dl}$, síntomas neuroglucopénicos (trastorno del sensorio y movimientos involuntarios) y la remisión de los síntomas al administrar soluciones glucosadas. En este momento debemos preguntarnos, ¿los síntomas que refiere el paciente (movimientos involuntarios) podrían explicarse por hipoglicemia crónica? La respuesta es que es muy probable, ya que la hipoglicemia se puede manifestar con síntomas autonómicos como palpitaciones, temblor, ansiedad, sudoración, hambre y parestesias, y síntomas neuroglucopénicos como: cambios conductuales, confusión, convulsiones y pérdida de conciencia (1). Nuestro paciente tenía síntomas neuroglucopénicos; la aparición de los síntomas en la mañana luego de un ayuno prolongado y su desaparición con la ingesta de alimentos apoyan la sospecha. Es importante destacar que los síntomas se asocian al nivel de glicemia que tenga el paciente, esto podría explicar que no siempre el paciente tuvo síntomas de trastorno del sensorio ya que ingería alimentos al presentar los movimientos involuntarios y que en el último episodio el ayuno fue más prolongado presentándose el trastorno del sensorio.

Ahora, el siguiente paso es discutir la etiología de la hipoglicemia crónica de este paciente. Los enfoques son diversos, el que voy a enfocar es bastante práctico. La causa más frecuente de hipoglicemia se encuentra en pacientes diabéticos que usan insulina o fármacos secretagogos de insulina (2), este paciente no es diabético y no usa fármacos que puedan inducir hipoglicemia, tiene familiares diabéticos y siempre se debe considerar la toma involuntaria o subrepticia de estos fármacos por lo que idealmente se debería dosar sulfonilureas en plasma (3); de todas maneras, esta etiología es de baja probabilidad. La siguiente causa, es la hipoglicemia del paciente crítico; esta opción es fácilmente descartada pues el paciente no tiene enfermedad crítica como falla renal, hepática o cardiaca; tampoco tiene sepsis. Las deficiencias hormonales como el hipocortisolismo sea primario (Enfermedad de Addison) o secundario pueden producir hipoglicemia asociada a ayuno prolongada, estos pacientes presentan síntomas asociados como hiperpigmentación de piel y mucosas en el caso de la enfermedad de Addison, además, pueden tener episodios agudos de hipotensión y dolor abdominal y en los exámenes de laboratorio pueden presentar hiponatremia e hiperkalemia, el diagnostico se hace con la prueba de estimulación de ACTH y un cortisol muy bajo en ayunas es sugerente; nuestro paciente no tiene la presentación clínica ni laboratorial de hipocortisolismo.

Otra causa de hipoglicemia crónica recurrente y de ayunas es el hiperinsulinismo por insulinoma, el diagnostico se apoya en la clínica y en el dosaje durante un episodio de hipoglicemia (glucosa menor de $40 \mathrm{mg} / \mathrm{dl}$ ) de insulina, péptido $\mathrm{C}$, proinsulina y $\beta$-hidroxbutirato; una vez hecho el diagnóstico laboratorial se procede a realizar imágenes CT o RM de abdomen, también ultrasonido endoscópico. En nuestro paciente podría considerarse esta posibilidad; pero otros hallazgos del examen fisco y de la placa de tórax, hacen que esta posibilidad no sea la más probable. Por último, consideremos los tumores de células no Beta como causantes de hipoglicemia; típicamente producen hipoglicemia de ayuno como la del paciente, los tumores son de gran volumen y de origen epitelial o mesenquimatosos entre los cuales tenemos hepatoma, carcinoma adrenocortical, tumor fibroso solitario del tórax, entre otros (4). Estos tumores producen un factor similar a la insulina tipo 2 (IGF 2 en inglés), que es la que produce la hipoglicemia, habitualmente se encuentra una tasa de IGF 2 / IGF 1 elevado (5); el tratamiento del tumor y hasta la disminución de su tamaño ayudan a controlar la hipoglicemia.

Nuestro paciente tiene en el examen físico y en la palca de tórax hallazgos correspondientes a una masa intratorácica; por lo dicho anteriormente esta gran masa intratorácica si podría estar asociada a la hipoglicemia crónica de este paciente. Como se mencionó, los tumores de células no beta que se 
asocian a hipoglicemia son en su mayoría de origen mesenquimatoso (41\%) y epiteliales (43\%). La pregunta seria, de estos ¿cuáles se podrían localizar en el tórax?: 1) los de origen mesenquimatosos: el mesotelioma y el tumor fibroso solitario, y 2) los de origen epiteliales los carcinomas de pulmón (adenocarcinoma, escamoso, células grandes y células pequeñas). Las imágenes de los carcinomas de pulmón son periféricas en adenocarcinoma y en el de células grandes, suelen ser nodulares o múltiples focos difusos de consolidación; además, presentan linfoadenopatías mediastinales y derrame pleural; la imagen de nuestro paciente es muy diferente a esta descripción. El tumor carcinoide bronquial, es un tumor neuroendocrino de origen endobronquial que en las imágenes se reflejan como atelectasia o neumonitis post obstructiva, también puede haber nódulos periféricos con calcificaciones internas; la imagen de nuestro paciente tampoco es compatible con esta descripción.

Ahora, veamos los tumores mesenquimatosos: el mesotelioma tiene una imagen diferente que consiste en efusiones pleurales unilaterales, engrosamiento y placas pleurales con calcificaciones. El tumor fibroso solitario es un tumor que afecta la pleura, se presenta como una gran masa intratorácica de bordes bien definidos adyacente a la pleura o al diafragma, es un tumor que suele ser benigno en el $80 \%$ de los casos, sus manifestaciones son las propias de un tumor torácico: disnea, tos, hemoptisis y baja de peso, tiene manifestaciones paraneoplásicas como hipoglicemia, osteoartropatía hipertrófica, acropaquías y paquidermatitis (6). Estos tumores manifiestan niveles elevados de IGF2 o de la relación IGF2/IGF1; el diagnostico final es mediante biopsia, la que se realiza al momento de la resección del tumor, que puede ser curativa y revertir las manifestaciones paraneoplásicas. Nuestro paciente tiene un cuadro clínico compatible con este tumor, las imágenes del tórax y TAC son características; por lo tanto, el diagnóstico más probable es un tumor fibroso solitario con manifestaciones paraneoplásicas como hipoglicemia y paquidermato-periostitis; el diagnóstico y tratamiento deben confirmarse mediante una resección del tumor. El plan debe ser la medición de IGF 2 e IGF1, y cirugía de exéresis de la tumoración.

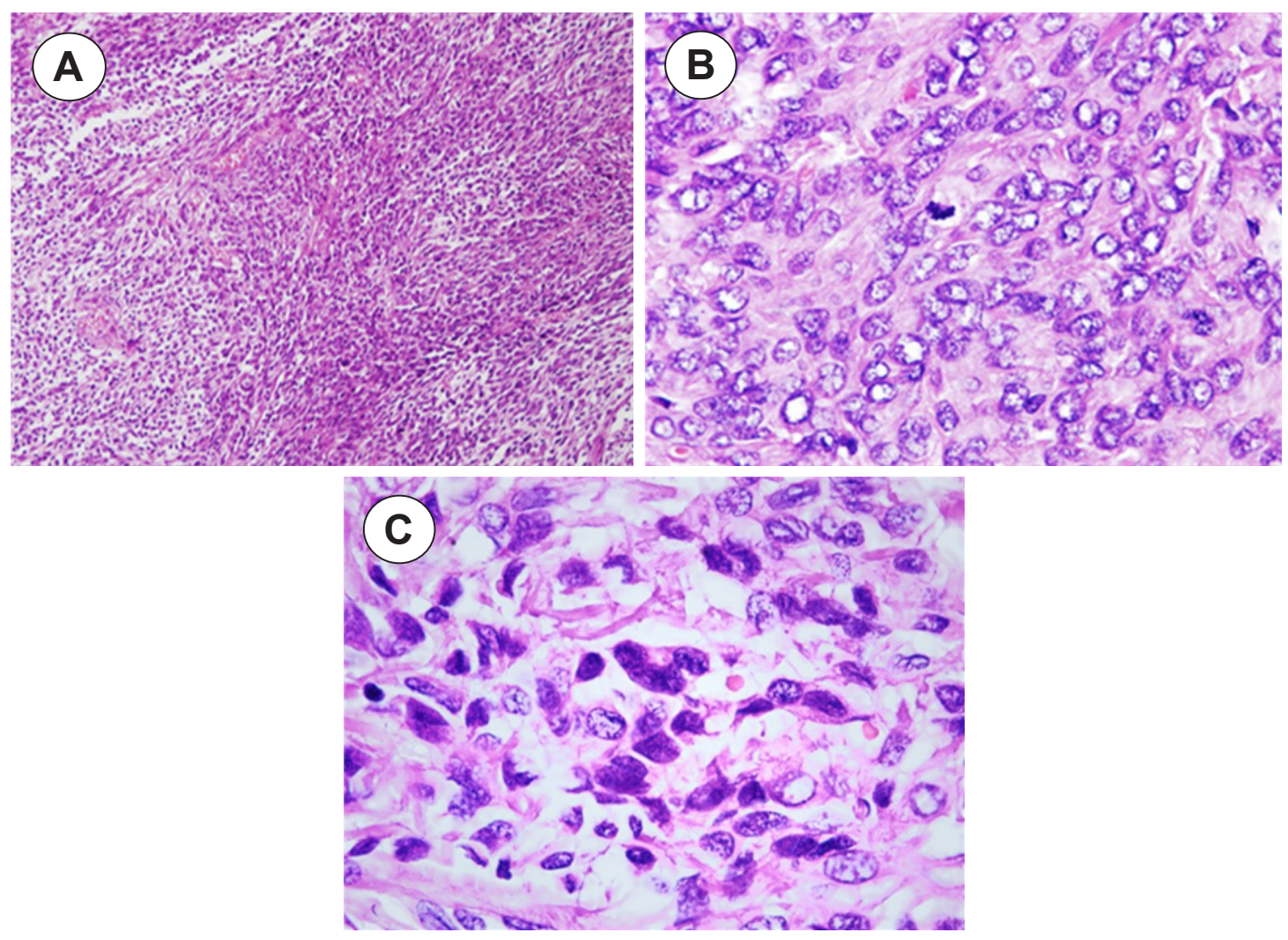

Figura 4. A: Áreas hipercelulares con células fusiformes dispuestas en fascículos. B: Células con atipia nuclear e incremento de la actividad mitótica (la presencia de $>4$ mitosis en 10 campos de alto poder es criterio diagnóstico para tumor fibroso solitario maligno). C: Citología atípica y el pleomorfismo nuclear. 


\section{Milton Blanco:}

Se procedió a la medición de IGF1 e IGF2, siendo sus resultados $41 \mathrm{ng} / \mathrm{ml}$ y $162 \mathrm{ng} / \mathrm{ml}$, respectivamente y la relación IGF2/IGF1 fue 4 (normal $<3$ ). Se procedió a la exéresis de la tumoración y lobectomía inferior derecha.

En la anatomía patológica se encontró: Tumoración nodular solida de $15 \times 5 \times 13 \mathrm{~cm}$, cubierta parcialmente por tejido fibroso, superficie con áreas congestivas y hemorrágicas. A las laminaciones, superficie de corte con áreas de aspecto edematoso, áreas de aspecto necrótico en $10 \%$ de la tumoración.

A la microscopía se observó proliferación de células fusiformes con patrón de crecimiento fascicular estoriforme con vasos hemangiopericitoides, que alternan con áreas hipocelulares hialinizadas. Las áreas hipercelulares presentan focos de hemorragia y necrosis con núcleos de tamaño mediano a grande. Áreas de mayor pleomorfismo y celularidad, hasta 4 mitosis en 10 campos de alto poder. Las células muestran positividad para CD34, BCL2, y son negativos para actina, s100, CD68, EMA, pan queratina, TTF1. Parénquima pulmonar y bronquio: no se observa infiltración por la neoplasia (Figura 4).

Diagnóstico Patológico: Tumor fibroso solitario

\section{Héctor Sosa:}

El diagnóstico fue confirmado con la biopsia completa del tumor con microscopia óptica e inmunohistoquímica. Como se mencionó anteriormente, la resección completa del tumor constituye el principal tratamiento. El riesgo de recurrencia es aproximadamente de 10 a $25 \%$ con una sobrevida a los 10 años de $73 \%$ (7).

La clasificación de "benigno" o "maligno" en este tumor es controversial ya que se basa en la histología y no se ha encontrado una relación directa entre esta clasificación y el pronóstico. Los criterios de alto riesgo de recurrencia son: la resección incompleta, metástasis en la presentación inicial, tumor de más de $10 \mathrm{~cm}$, tasa mitótica alta y necrosis en el tejido (8). Tanto la quimioterapia como la radioterapia se deben contemplar en la evaluación caso por caso y no se recomiendan si la resección ha sido completa (9). El seguimiento para recurrencia se basa en la descripción del tumor (sésil o pedunculado) y en si tiene riesgo alto de recurrencia, y se hace con imágenes. Nuestro paciente tiene criterios de alto riesgo por el tamaño de la masa, la tasa mitótica y la presencia de necrosis, por lo que amerita seguimiento cercano.

\section{EVOLUCIÓN}

Luego de la cirugía no se evidenciaron nuevos eventos de hipoglicemia ni de síntomas asociados. La paquidermato-periostitis remitió también luego de la cirugía (Figura 5 y 6 ).

\section{DIAGNÓSTICO FINAL}

Hipoglicemia paraneoplásica y paquidermato periostitis por tumor fibroso solitario torácico.

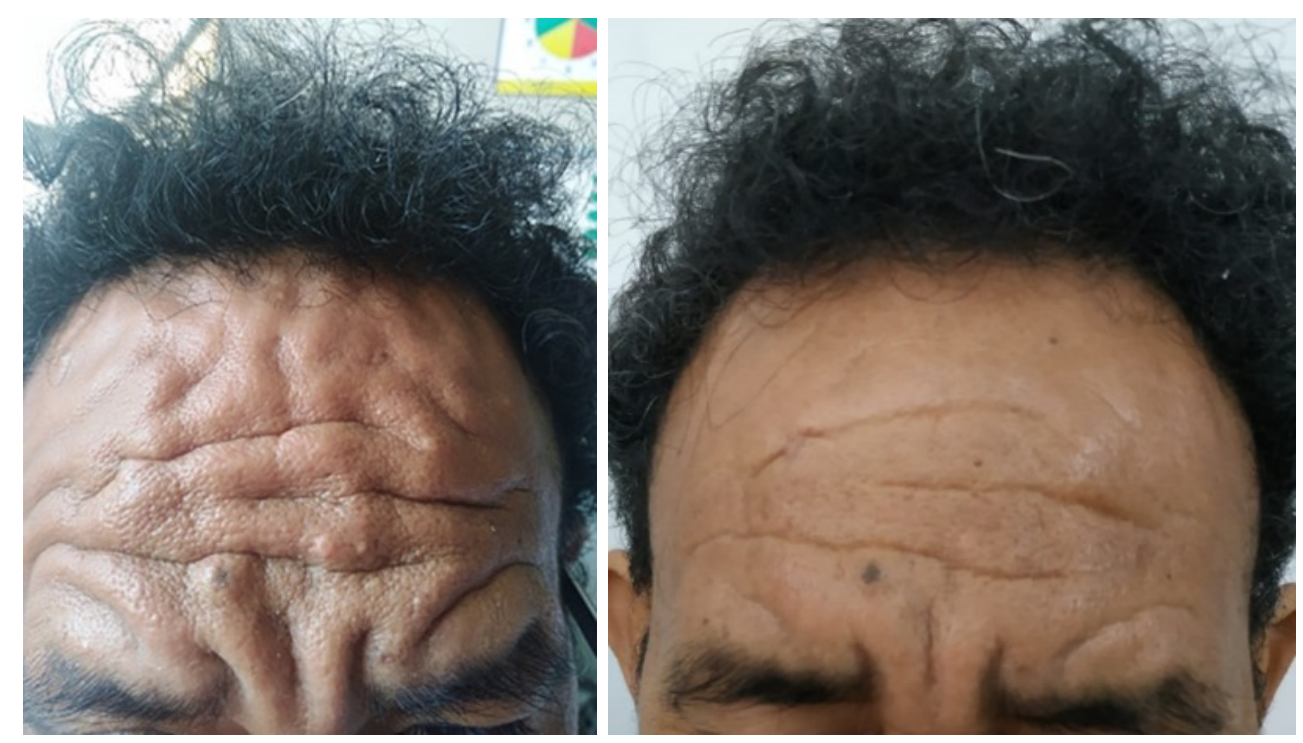

Figura 5. Reversión de cambios cutáneos. 

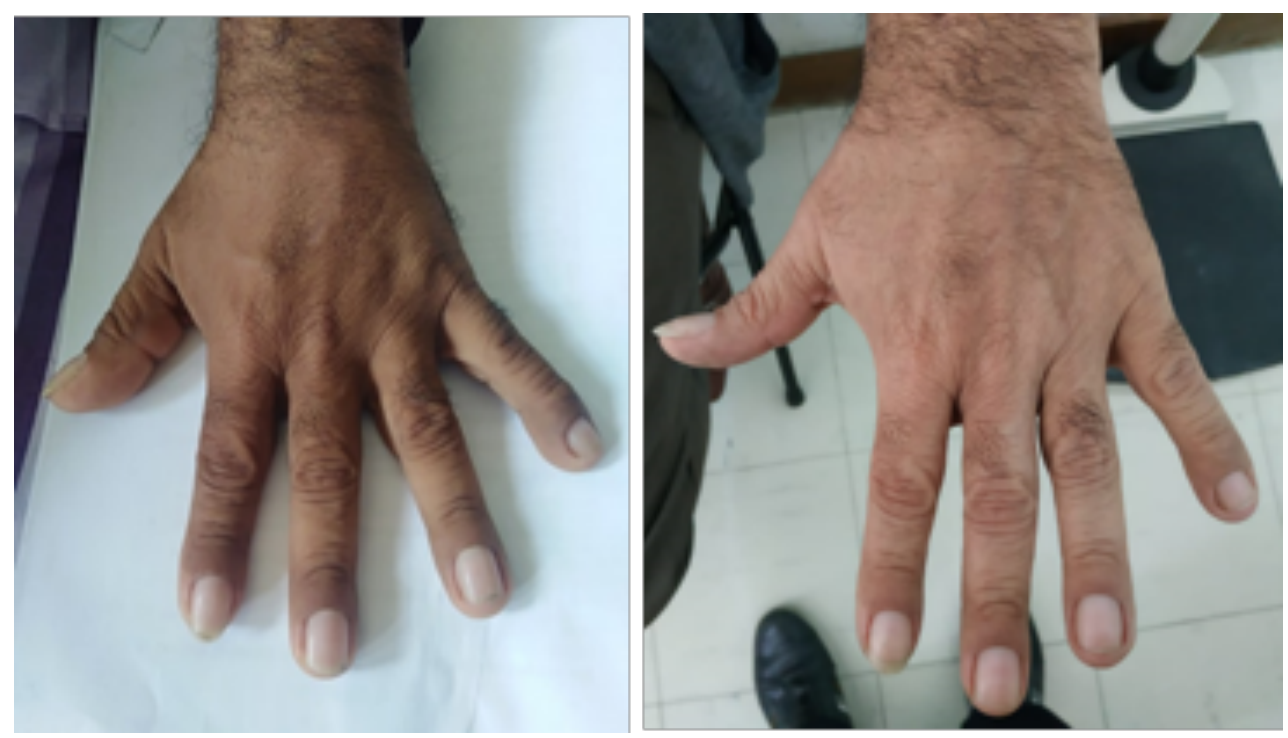

Figura 6. Reversión de acropaquías.

\section{REFERENCIAS BIBLIOGRÁFICAS}

1. Service FJ, Diagnostic approach to adults with hypoglycemic disorders. Endocrinol Metab Clin North Am. 1999; 28(3):519-32.

2. Cryer PE, Axelrod L, Grossman AB, et al. Evaluation and management of adult hypoglycemic disorders: An Endocrine Society Clinical Practice Guideline. J Clin Endocrinol Metab. 2009; 94:709-28.

3. Perros P, Henderson AK, Carter DC, et al. Lesson of the week. Are spontaneous hypoglycaemia, raised plasma insulin and $\mathrm{C}$ peptide concentrations, and abnormal pancreatic images enough to diagnose insulinoma? BMJ. 1997; 314(7079):496-7.

4. Scott K. Non-islet cell tumor hypoglycemia. J Pain Symptom Manage. 2009; 37(4):e1-3.. doi: 10.1016/j. jpainsymman.2008.11.004.
5. Dynkevich Y, Rother KI, Whitford I, et al. Tumors, IGF-2, and hypoglycemia: insights from the clinic, the laboratory, and the historical archive. Endocr Rev. 2013 Dec; 34(6):798-826.

6. Jang JG, Chung JH, Hong KS, et al. A case of solitary fibrous pleura tumor associated with severe hypoglycemia: doege-potter syndrome. Tuberc Respir Dis (Seoul). 2015 Apr; 78(2):120-4.

7. Demicco EG, Park MS, Araujo DM, et al. Solitary fibrous tumor: a clinicopathological study of 110 cases and proposed risk assessment model. Mod Pathol. 2012 Sep; 25(9):1298-306.

8. Van Houdt WJ, Westerveld CM, Vrijenhoek JE, et al. Prognosis of solitary fibrous tumors: a multicenter study. Ann Surg Oncol. 2013 Dec; 20(13):4090-5.

9. De Perrot M, Fischer S, Bründler MA, et al. Solitary fibrous tumors of the pleura. Ann Thorac Surg. 2002; 74(1):285-93. 